\title{
DOA estimation with synthetic nested array using moving sensor
}

\author{
Zhan Shi ${ }^{1, a}$, Xiaofei Zhang ${ }^{1,2,3, b}$, You Sun ${ }^{1, c}$ \\ ${ }^{1}$ College of Electronic and Information Engineering, Nanjing University of Aeronautics and \\ Astronautics, Nanjing, 210016, People's Republic of China \\ ${ }^{2}$ State Key Laboratory of Millimeter Waves, Southeast University, Nanjing, China, 210096. \\ ${ }^{3}$ National Mobile Communications Research Laboratory, Southeast University, Nanjing, China, \\ 210096. \\ a'Sz_yz@163.com, bzhangxiaofei@nuaa.edu.cn, c sunyou_nuaa@163.com
}

Keywords: Moving sensor, Nested array, Synthetic array.

\begin{abstract}
.
The synthetic aperture methods are widely used to extend array aperture by exploiting platform motion. In this paper, a synthetic nested array using moving sensor for DOA estimation is proposed. With the moving sensor, we can construct a longer nested array which is equivalent to the real array. Then traditional DOA estimation algorithms for nested array can also be applied, such as spatial smooth MUSIC algorithm. By extending the array aperture and exploiting synthetic nested array, the DOA estimation performance can be improved. In the ideal case, the performance of synthetic and real array is the same which is vilified by performance analysis and simulations.
\end{abstract}

\section{Introduction}

Direct of arrival (DOA) estimation with high resolution is a fundamental subject in array signal processing. To improve the DOA resolution we have to enlarge the array aperture which increases the load on the moving platform. Therefore, methods of extending the aperture are widely researched, such as virtual array expansion algorithms [1-2], data reconstruction method [3] and forth-order cumulants methods [4-5]. But these methods are to further explore the information of received signals and do not really extend the array aperture.

Synthetic aperture technology is widely used in synthetic aperture sonar [6] and synthetic aperture radar [7], which exploits the mobility of the platform and uses the temporal signal coherence to extend the array aperture by signal processing [8-9]. Inspired by the synthetic aperture technology, we can use the moving array to synthesize a virtual array to expand the array aperture.

This paper combines the moving array with the nested array [10] and proposes a DOA estimation method with the synthetic nested array using moving sensor. Firstly, the signal model of the moving single sensor is established by selecting the appropriate sampling time interval, then the equivalent nested array is constructed and the DOA estimation is performed using the spatial smooth MUSIC (SS-MUSIC) method. To facilitate the performance analysis and mainly to compare the difference between the synthetic and real array method, we do not consider the effect of the signal model error.

The rest of this paper is organized as follows. The data models of single moving sensor and nested array are presented in Section 2. In Section 3, we propose the DOA estimation method with synthetic nested array using moving sensor. Section 4 analyzes the performance of the synthetic array while numerical simulations are provided in Section 5. At last, Section 6 concludes the paper.

\section{Data Models}

First, we assume 1) the received signals are far-field monochromatic narrowband signals and the 
signals of different sources are not relevant; 2) the angles do not change during the synthetic period; 3) the time needed for synthesis is less than the maximum correlation time of the signal; 4) signal frequency and sensor's velocity are known.

2.1 Moving array model. Consider a single sensor moves linearly along the $\mathrm{x}$-axis in the XOY plane with the constant velocity $v$, where $v$ is known. A far-field monochromatic signal $s(t)$ impinge on the sensor with the angle $\theta$, as shown in Fig 1 . The signal is sampled with time interval $\tau$, and the received signal of the sensor at time $t_{n}\left(t_{n}=n \tau, n=1,2, \cdots\right)$ can be expressed as

$$
x\left(t_{n}\right)=s\left(t_{n}\right)+\varepsilon\left(t_{n}\right)=A_{m} \exp \left[j 2 \pi f\left(1+\frac{v \sin \theta}{c}\right) t_{n}\right]+\varepsilon\left(t_{n}\right),
$$

where $A_{m}$ is the signal amplitude which is considered to be constant in the synthetic period; $f$ and $c$ stand for signal frequency and velocity of light respectively; $\varepsilon\left(t_{n}\right)$ is the additive Gaussian white noise received at time $t_{n}$ and is independent of the signal with zero mean and variance $\sigma^{2}$.

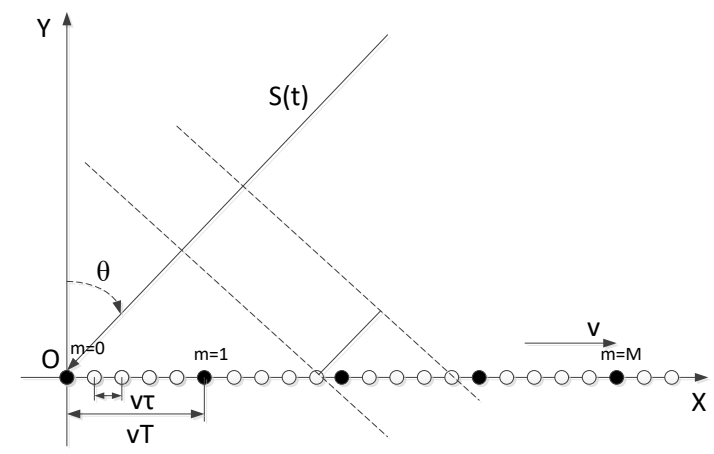

Fig.1 Moving single sensor model

2.2 Nested array model. Consider a two-level nested array with $N$ sensors which consists of two ULAs. The inner ULA has $N_{1}$ elements with spacing $d_{1}=d$ and the outer has $N_{2}$ elements with spacing $d_{2}=\left(N_{1}+1\right) d$. The sensor locations are given by the set $\Phi=\left\{n_{i} d, i=1,2, \ldots, N\right\}$, where $n_{i}$ is integer and $\left\{n_{i}=0,1, \ldots, N_{1}-1, N_{1}, \ldots, N_{2}\left(N_{1}+1\right)-1\right\}$. Here we take the first element of inner ULA as reference. Fig 2 depicts a two-level nested array with $N_{1}=N_{2}=3$.

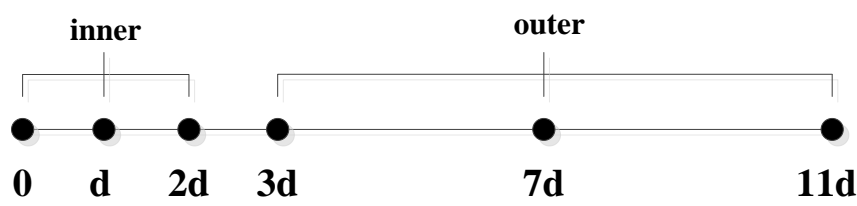

Fig.2 Two-level nested array model

Assume $K$ far-field narrowband sources impinging on the nested array from the angle $\theta_{i}(i=1,2, \ldots, K)$ with power $\sigma_{i}^{2}(i=1,2, \ldots, K)$, then the received signals can be expressed as

$$
\mathbf{x}(t)=\mathbf{A s}(t)+\boldsymbol{\varepsilon}(t)
$$

where $\mathbf{A}=\left[\mathbf{a}\left(\theta_{1}\right), \mathbf{a}\left(\theta_{2}\right), \ldots, \mathbf{a}\left(\theta_{K}\right)\right] \in \mathbb{C}^{N \times K}, \mathbf{a}\left(\theta_{i}\right)=\left[e^{j 2 \pi d_{1} \sin \theta_{i} / \lambda}, e^{j 2 \pi d_{2} \sin \theta_{i} / \lambda}, \ldots, e^{j 2 \pi d_{N} \sin \theta_{i} / \lambda}\right]^{T}$ stands for the steering vector for the $i$-th target; $\lambda$ is the wavelength and $d_{i} \in \Phi$ is the sensor location; $\mathbf{s}(t)=\left[s_{1}(t), s_{2}(t), \ldots, s_{K}(t)\right]^{T} \in \mathbb{C}^{K \times 1} ; \boldsymbol{\varepsilon}(t) \in \mathbb{C}^{N \times 1}$ is the white Gaussian noise vector. 


\section{DOA estimation method with synthetic nested array using moving sensor}

Define the unit sampling period $T=L \tau$, intercept $L$ sample points of signal received by the moving sensor at time $T_{i}(i=1,2, \ldots N)$, and construct the data matrix

$$
\mathbf{X}=\left[\begin{array}{cccc}
x\left(t_{0}+T_{1}\right) & x\left(t_{0}+T_{1}+\tau\right) & \cdots & x\left(t_{0}+T_{1}+(L-1) \tau\right) \\
x\left(t_{0}+T_{2}\right) & x\left(t_{0}+T_{2}+\tau\right) & \cdots & x\left(t_{0}+T_{2}+(L-1) \tau\right) \\
\vdots & \vdots & \ddots & \vdots \\
x\left(t_{0}+T_{N}\right) & x\left(t_{0}+T_{N}+\tau\right) & \ldots & x\left(t_{0}+T_{N}+(L-1) \tau\right)
\end{array}\right],
$$

where $x\left(t_{0}\right)$ is the reference signal, $T_{i}=n_{i} T$. Each row of data matrix $\mathbf{X} \in \mathbb{C}^{N \times L}$ represents $L$ sample points during the period $T$ and the number of rows stands for the number of data segments.

According to the data model in (1), we can obtain the following equation while ignore the noise

$$
x\left(t_{0}+T_{n}+(l-1) \tau\right)=e^{j \omega\left(T_{n}-T_{n-1}\right)} x\left(t_{0}+T_{n-1}+(l-1) \tau\right), n=1,2, \ldots, N, l=1,2, \ldots, L,
$$

where $\omega=2 \pi f(1+v \sin \theta) / c ; x\left(t_{0}+T_{n}+(l-1) \tau\right)$ and $x\left(t_{0}+T_{n-1}+(l-1) \tau\right)$ are the elements of $n$-th row $l$-th column and ( $n$ - 1 )-th row $l$-th column in $\mathbf{X}$ respectively. That is, the corresponding elements of the received signals in two adjacent rows have the same phase difference $e^{j \omega\left(T_{n}-T_{n-1}\right)}(n=2, \ldots, N)$. It can be seen that the received signal matrix here is similar to the received signal matrix of the real array: the elements of each column in $\mathbf{X}$ are equivalent to the snapshots of the real array, and each row of $\mathbf{X}$ is equivalent to the signals received by one sensor, which means the number of rows of $\mathbf{X}$ is equivalent to the number of sensors in the real array. In general, we use the data received by one single sensor constructing a data matrix which is equivalent to the data matrix received by a longer real array. This process is called moving array synthetic process.

The $l$-th column of $\mathrm{X}$ can be represented as

$$
\mathbf{x}(l)=\mathbf{a}(\varphi) s\left(t_{0}+T_{1}+(l-1) \tau\right)+\mathbf{\varepsilon}(l), l=1,2, \ldots, L,
$$

where $\mathbf{a}(\varphi)=\left[1, e^{j 2 \omega\left(T_{2}-T_{1}\right)}, \ldots, e^{j \omega\left(T_{N}-T_{N-1}\right)}\right]^{T}$.

Popularize the single source case above to multi-sources case. Assume $K$ far-field narrowband sources impinging on the moving array from the angle $\theta_{i}(i=1,2, \ldots, K)$ with power $\sigma_{i}^{2}(i=1,2, \ldots, K)$, and (5) can be expressed as

$$
\mathbf{x}(l)=\mathbf{A S}\left(t_{0}+T_{1}+(l-1) \tau\right)+\boldsymbol{\varepsilon}(l), l=1,2, \ldots, L,
$$

where $\mathbf{A}=\left[\mathbf{a}\left(\theta_{1}\right), \ldots \mathbf{a}\left(\theta_{K}\right)\right] \in \mathbb{C}^{N \times K}, \mathbf{a}\left(\theta_{i}\right)=\left[1, e^{j \omega_{i}\left(T_{2}-T_{1}\right)}, \ldots, e^{j \omega_{i}\left(T_{N}-T_{N-1}\right)}\right]^{T}, \omega_{i}=2 \pi f\left(1+v \sin \theta_{i}\right) / c \quad$, $\mathbf{S}\left(t_{0}+T_{1}+(l-1) \tau\right)=\left[s_{1}\left(t_{0}+T_{1}+(l-1) \tau\right), s_{2}\left(t_{0}+T_{1}+(l-1) \tau\right), \ldots, s_{K}\left(t_{0}+T_{1}+(l-1) \tau\right)\right]^{T} \in \mathbb{C}^{K \times 1}$.

Fig.3 depicts an example of moving synthetic array, where each black solid point represents the first sample point for each segment, which is equivalent to the sensor position of the real array. The hollow points represent the sample points in each segment and are equivalent to the snapshots. The distance $v T_{i}(i=1,2, \ldots, N)$ is just like the spacing $d_{i}$ in the real array.

If let $v T=d$, it can be seen that with the assumption $T_{i}=n_{i} T$, we can obtain $v T_{i}=n_{i} v T=n_{i} d$, which means the synthetic array model is similar to the real nest array model mentioned above, and the DOA algorithms for the nested array can be applied to the synthetic array. 


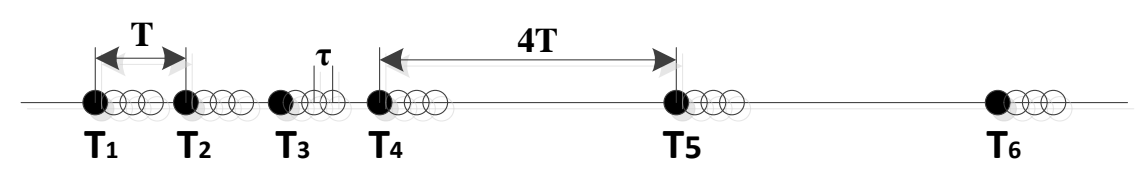

Fig.3 An example of moving synthetic array

The covariance matrix of (3) can be expressed as

$$
\mathbf{R}_{x}=E\left[\mathbf{X} \mathbf{X}^{H}\right]=\mathbf{A} \mathbf{R}_{s} \mathbf{A}^{H}+\sigma_{n}^{2} \mathbf{I}
$$

where $\mathbf{R}_{s}=\operatorname{diag}\left[\sigma_{1}^{2}, \sigma_{2}^{2}, \ldots, \sigma_{K}^{2}\right]$.

Vectorize the covariance matrix in (7) and the observing vector can be represented by

$$
\begin{aligned}
\mathbf{z} & =\operatorname{vec}\left(\mathbf{R}_{x}\right)=\operatorname{vec}\left[\sum_{i=1}^{K} \sigma_{i}^{2} \mathbf{a}\left(\varphi_{i}\right) \mathbf{a}^{H}\left(\varphi_{i}\right)\right]+\sigma_{n}^{2} \mathbf{e}=\left(\mathbf{A}^{*} \odot \mathbf{A}\right) \mathbf{p}+\sigma_{n}^{2} \mathbf{e} \\
& =\mathbf{B} \mathbf{p}+\sigma_{n}^{2} \mathbf{e},
\end{aligned}
$$

where $\mathbf{e}=\left[\mathbf{e}_{1}^{T}, \mathbf{e}_{2}^{T}, \ldots, \mathbf{e}_{N}^{T}\right]^{T}, \mathbf{e}_{i} \in \mathbb{R}^{N \times 1}$ is a vector consisting of zeros except a number 1 at the $i$-th position; $\mathbf{p}=\left[\sigma_{1}^{2}, \sigma_{2}^{2}, \ldots, \sigma_{K}^{2}\right]^{T}$ and $\mathbf{B}=\mathbf{A}^{*} \odot \mathbf{A}$, where $\odot$ stands for Khatri-Rao product,.

The dimension of the new manifold $\mathbf{B}$ is $N^{2} \times K$, but the number of distinct rows is equal to the DOFs of the difference co-array. After removing the repeated rows from $\mathbf{z}$ and sorting left distinct rows corresponding to the location in the difference co-array, we can get a new observation vector

$$
\mathbf{z}_{1}=\mathbf{B}_{1} \mathbf{p}+\sigma_{n}^{2} \mathbf{e}^{\prime}
$$

where $\mathbf{B}_{1}$ is the new manifold after removing the repeat rows from manifold $\mathbf{B}, \quad \mathbf{e}^{\prime} \in \mathbb{R}^{\left(N^{2} / 2+N-1\right) \times 1}$ is a vector of all zeros except a 1 at the $\left(N^{2} / 4+N / 2\right)$-th position. In (9), $\mathbf{p}$ is the equivalent source for difference co-array, but it consists of $K$ actual sources with power $\sigma_{i}^{2}$ so they behave like fully coherent sources. As the difference co-array of the two-level nested array is a filled ULA, we can use spatial smooth MUSIC (SS-MUSIC) method for DOA estimation.

According to [10], we divide the ( $N^{2} / 2+N-1$ )-elements ULA into $N^{2} / 4+N / 2$ overlapping subarrays and each subarray has $N^{2} / 4+N / 2$ elements. The $i$-th subarray has sensors located at $\left\{(-i+1+n) d, n=0,1, \ldots, N^{2} / 4+N / 2-1\right\}$ and we denote the following full rank covariance matrix

$$
\mathbf{R}_{z}=\frac{1}{N^{2} / 4+N / 2} \sum_{i=1}^{N^{2} / 4+N / 2} \mathbf{z}_{1 i} \mathbf{z}_{1 i}^{H},
$$

where $\mathbf{z}_{1_{i}}$ consists of the $\left(N^{2} / 4+N / 2-i+1\right)$-th to $\left(N^{2} / 2+N-i+1\right)$-th rows of $\mathbf{z}_{1}$. Then DOA estimation can be obtained through searching the SS-MUSIC spectrum as follow

$$
P(\theta)=\frac{1}{\mathbf{a}^{H}(\hat{\theta}) \mathbf{U}_{n} \mathbf{U}_{n}^{H} \mathbf{a}(\hat{\theta})},
$$

where $\mathbf{a}(\theta)=\left[1, e^{j 2 \pi f(1+v \sin \hat{\theta} / c) T}, \ldots, e^{j 2 \pi f(1+v \sin \hat{\theta} / c)\left(N^{2} / 4+N / 2-1\right) T}\right]^{T}, \hat{\theta} \in(-\pi / 2, \pi / 2) ; \quad \mathbf{U}_{n}$ stands for noise subspace of covariance matrix $\mathbf{R}_{z}$. 


\section{Performance analysis}

Cramer-Rao bound (CRB) [12] for phase $\Omega$ can be represented as

$$
C R B(\Omega)=\frac{\sigma^{2}}{2}\left\{\sum_{i=1}^{L} \operatorname{Re}\left[\mathbf{S}^{H}(i) \mathbf{D}\left(\mathbf{I}-\mathbf{A}\left(\mathbf{A}^{H} \mathbf{A}\right)^{-1} \mathbf{A}^{H}\right) \mathbf{D}^{H} \mathbf{S}(i)\right]\right\}^{-1},
$$

where $\mathbf{S}(i)=\operatorname{diag}\left[s_{1}(i), s_{2}(i), \ldots, s_{K}(i)\right], \mathbf{D}=\left[\mathbf{d}\left(\Omega_{1}\right), \mathbf{d}\left(\Omega_{2}\right), \ldots, \mathbf{d}\left(\Omega_{K}\right)\right], \mathbf{d}\left(\Omega_{k}\right)=d \mathbf{a}\left(\Omega_{k}\right) / d \Omega_{k}$.

If the frequency is known, then CRB for angle $\theta_{k}$ can be expressed as [13]

$$
\operatorname{CRB}\left(\theta_{k}\right)=\operatorname{CRB}\left(\Omega_{k}\right)\left(\frac{d \theta_{k}}{d \Omega_{k}}\right)^{2} \text {. }
$$

where for a synthetic nested array $\Omega_{v k}=2 \pi f\left(1+v \sin \theta_{k} / c\right) T_{i}=2 \pi f T_{i}+2 \pi f \cdot v T_{i} \sin \theta_{k} / c$ and for a real nested array $\Omega_{r k}=2 \pi f d_{i} \sin \theta_{k} / c$. It can be seen that if let $d_{i}=v T_{i}$, then the phase difference of the real array and the synthetic array is equivalent except a constant factor $2 \pi f T_{i}$ which does not contain DOA information. Therefore models for the real and synthetic array are consistent when analyze the deviation of phase difference $\Omega_{r k}$ and $\Omega_{v k}$. For simplicity let $2 \pi f=\omega$.

From (12) (13), the CRB for angle $\theta_{k}$ for a real array is

$$
\operatorname{CRB}_{r}\left(\theta_{k}\right)=\operatorname{CRB}\left(\Omega_{r k}\right)\left(\frac{d \theta_{k}}{d \Omega_{r k}}\right)^{2}=\operatorname{CRB}\left(\Omega_{r k}\right) \cdot \frac{c^{2}}{\omega^{2} d_{i}^{2}-\Omega_{r k}^{2} c^{2}},
$$

and for a synthetic array is

$$
C R B_{v}\left(\theta_{k}\right)=\operatorname{CRB}\left(\Omega_{v k}\right)\left(\frac{d \theta_{k}}{d \Omega_{v k}}\right)^{2}=\operatorname{CRB}\left(\Omega_{v k}\right) \cdot \frac{c^{2}}{\omega^{2} v^{2} T_{i}^{2}-\left(\Omega_{v k}-\omega T_{i}\right)^{2} c^{2}} .
$$

As models for the real array and the synthetic array are consistent when analyze the deviation of phase difference $\Omega_{r k}$ and $\Omega_{v k}$, and under the assumption $d_{i}=v T_{i}$, we can get that CRB for corresponding real array and synthetic array is the same. It means in the ideal case the synthetic nested array using moving sensor proposed above can have the same DOA estimation performance with the corresponding real nested array.

\section{Simulation results}

In this section, numerical simulations are provided to validate the effectiveness of the proposed synthetic nested array using moving sensor. For the real nested array, $N=N_{1}+N_{2}=3+3=6$ and for the synthetic array, we intercept 6 data segments for fair comparison. Then two nested array can resolve $N^{2} / 4+N / 2-1=11$ targets at most. We assume that there are 9 targets from angle [- $40^{\circ}$, $-30^{\circ},-20^{\circ},-10^{\circ}, 0^{\circ}, 10^{\circ}, 20^{\circ}, 30^{\circ}, 40^{\circ}$ ] impinging on the array, the frequency $f=1 \mathrm{GHz}$, the velocity of moving sensor $v=100 \mathrm{~m} / \mathrm{s}$ and the period $T=d / v=\lambda / 2 v=1.5 \mathrm{~ms}$. As the performance metric, root mean square error (RMSE) is employed, i.e.

$$
R M S E=\sqrt{\frac{1}{K} \frac{1}{M} \sum_{i=1}^{M} \sum_{i=1}^{K}\left(\theta_{k}-\hat{\theta}_{k m}\right)^{2}},
$$

where $M$ is the number of Monte-Carlo simulations and $\hat{\theta}_{k m}$ stands for the $m$-th trial estimation of the $k$-th angle $\theta_{k}$. For all the following simulations, we set $M=1000$. 
5.1 MUSIC spectrum. Fig.4 depicts the spectrum by exploiting SS-MUSIC algorithm with synthetic and real nested array, where $\mathrm{SNR}=10 \mathrm{~dB}$ and $L=500$, for the real nested array $L$ means snapshots while for the synthetic nested array $L$ stands for the number of sample points in one data segment. It is clearly that both two arrays can detect all targets.

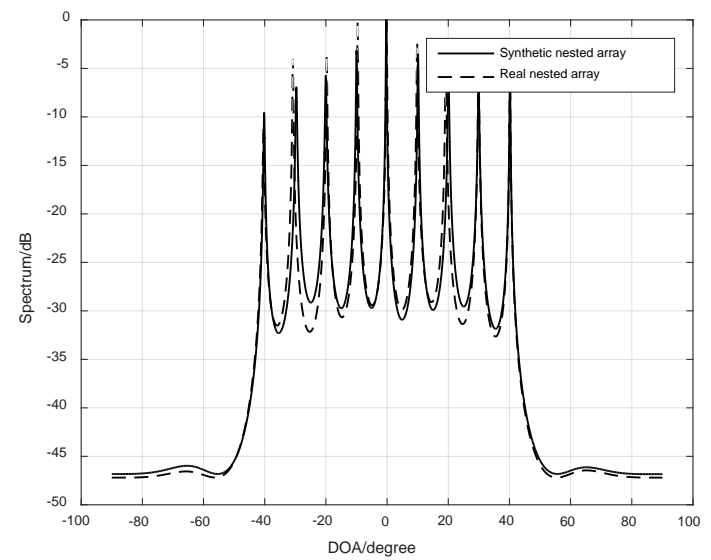

Fig. 4 Spatial spectrum with SS-MUSIC using synthetic and real nested array

5.2 RMSE performance of different arrays. We employ SS-MUCIC to study the RMSE performance of DOA estimation with the proposed synthetic nested array, real nested array [11] and synthetic ULA [9]. As there are 9 targets impinging on the array, to detect all the targets the synthetic ULA need 10 segments at least which is similar to a real ULA with 10 sensors. Thus, for the real and synthetic nested array $L=500$, and for synthetic ULA $L^{\prime}=N L / 10=6 \times 500 / 10=300$. Fig.5 captures the RMSE performance using SS-MUSIC with the three arrays above. We can get that the synthetic and real nested array have almost the same performance which corresponds to the analysis in the section above and the nested arrays perform better than the synthetic ULA when the number of data is the same, due to the nested arrays can provide more DOFs.

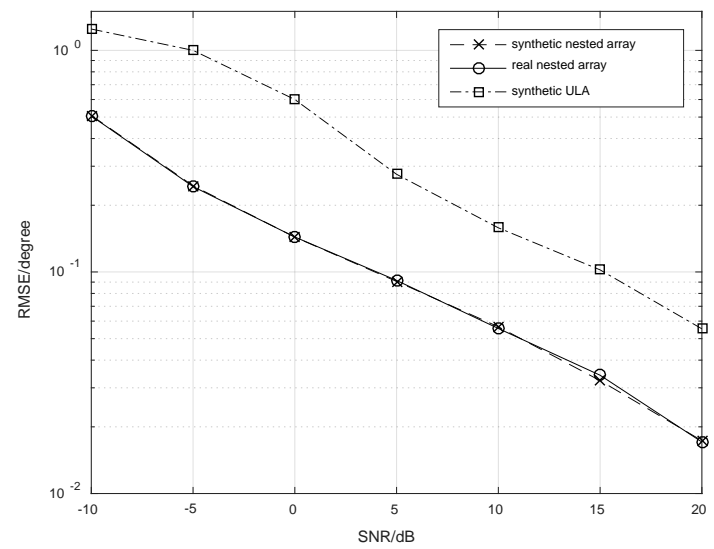

Fig.5 RMSE performance of synthetic and real nested arrays versus SNR

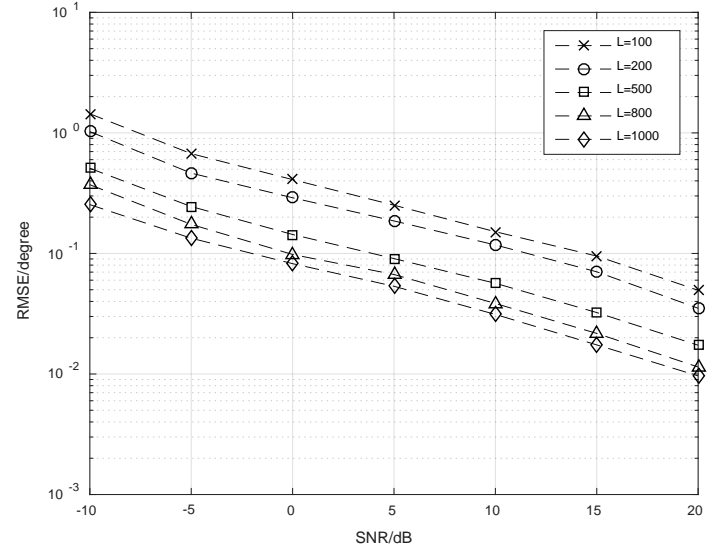

Fig.6 RMSE performance of synthetic nested array versus SNR and snapshots

5.3 RMSE performance versus SNR and snapshots. Fig.6 illustrates performance of the synthetic nested array versus SNR and snapshots. It is shown that the performance of synthetic nested array improves with the SNR and snapshots increasing. As we assume that the time needed for synthesis is less than the maximum correlation time of the signal, so the more snapshots we use the better performance will be, but if the assumption does not hold estimation performance will get worse. The minimum temporal coherence period needed for synthesis is discussed in [14]. 


\section{Conclusion}

In this paper, a synthetic nested array using moving sensor is proposed to extend the array aperture and improve DOA estimation performance. With only one single moving sensor, an equivalent synthetic nested array is formed by constructing a data matrix which is similar to the one in real arrays with sample signals at a serious of defined time. Then the traditional DOA estimation algorithm for nested arrays can be exploited, such as SS-MUSIC. In the ideal case, the performance of synthetic and real array is the same which is vilified by performance analysis and simulations.

\section{Acknowledgement}

This work is supported by China NSF Grants (61371169, 61601167), the open research fund of National Mobile Communications Research Laboratory, Southeast University (No.2015D030), JiangSu NSF (BK20161489), the open research fund of State Key Laboratory of Millimeter Waves, Southeast University(No.K201826), and the Fundamental Research Funds for the Central Universities (No:NE2017103).

\section{References}

[1] Chevalier P, Albera L, Ferreol A, et al. On the virtual array concept for higher order array processing[J]. IEEE Transactions on Signal Processing, 2005, 53(4):1254-1271.

[2] Osman A, Noureldin A. Direction of arrival estimation using virtual array search[J]. Iet Radar Sonar \& Navigation, 2011, 5(4):389-397.

[3] Li S, Yang X, Jiang X, et al. Direction-of-Arrival Estimation Based on Khatri-Rao Product and Redundancy Arrays[J]. International Journal of Signal Processing Image Processing \& P, 2014,

[4] Ye Z, Zhang Y. DOA estimation for non-Gaussian signals using fourth-order cumulants[J]. Iet Microwaves Antennas \& Propagation, 2009, 3(7):1069-1078.

[5] Xiang L, Ye Z, Xu X, et al. Direction of arrival estimation for uniform circular array based on fourth-order cumulants in the presence of unknown mutual coupling[J]. Iet Microwaves Antennas \&Propagation, 2008, 2(3):281-287.

[6] Wachowski N, Azimi-Sadjadi M R. A New Synthetic Aperture Sonar Processing Method Using Coherence Analysis[J]. IEEE Journal of Oceanic Engineering, 2011, 36(4):665-678.

[7] Wiley C A. Synthetic Aperture Radars[J]. IEEE Transactions on Aerospace \& Electronic Systems,1985, AES-21(3):440-443.

[8] Autrey S W. Passive synthetic arrays[J]. Journal of the Acoustical Society of America, 1988, 84(84):592-598.

[9] Sullivan E J. On the role of modeling in passive synthetic aperture processing[C]// Oceans. IEEE,2000:7-9 vol.1.

[10] Pal P, Vaidyanathan P P. Nested Arrays: A Novel Approach to Array Processing With Enhanced Degrees of Freedom[J]. IEEE Transactions on Signal Processing, 2010, 58(8):4167-4181.

[11] Ma W K, Hsieh T H, Chi C Y. DOA estimation of quasi-stationary signals via Khatri-Rao subspace[C]// IEEE International Conference on Acoustics. IEEE,2009:2165-2168.

[12] Stoica P, Arye N. MUSIC, maximum likelihood, and Cramer-Rao bound[J]. IEEE Transactions on Acoustics Speech \& Signal Processing, 1989, 37(5):720-741.

[13] Wang Jianpeng, Liu Zheng, Jiang Wenli. DOA estimation for passive arrays of moving single sensor[J]. Acta Aeronautica ET Astronautica Sinica, 2010,31(7):1445-1453.

[14] Ramirez J, Krolik J. Multiple source localization with moving co-prime arrays[C]// IEEE International Conference on Acoustics, Speech and Signal Processing. IEEE, 2015:2374-2378. 